\title{
Sistemas electorales en El Salvador: El caso de las elecciones de diputados $y$ de concejos municipales
}

$\square$ n 1993, de cara a las llamadas "elecciones del siglo" de Marzo de 1994, incursionabamos en el esludio de los sistemas electorales utilizados en EI Salvador con el objeto de encontrar una clave para comprender cómo éstos no son meros instrumentos técnicos para transformar los votos en escaños sino verdaderos marcos institucionales que favorecen a unos partidos en detrimento de otros y que, por tanto, una reforma electoral tampoco es una cuestión puramente técnica sino que es también un asunto político (Artiga-González, 1993).

Hoy otra vez estamos en un año pre-electoral. Sin embargo, si las "elecciones del siglo" no generaron mayores expectativas en buena parte de la población salvadoreña con capacidad de voto, a pesar del momento transicional de 1994, tiene sentido no esperar gran atención sobre el evento elecloral de 1997. Tómese en cuenta además que las próximas elecciones poseen, de por sí, una menor importancia dado el régimen presidencialista salvadoreño ya que en éste son las elecciones de presidente y vice-presidente las que captan la mayor atención. ¿Por qué entonces escribir sobre sistemas electorales en este momento? En primer lugar, para dar a conocer parte de los hallazgos de una investigación hecha por nosotros entre 1994 y 1996 (Artiga-González, 1996). En segundo lugar, para contribuir, mediante su divulgación, al desarrollo de los estudios políticos en El Salvador. Y, en tercer lugar, para lanzar algunas ideas en tomo a lo no considerado por la reforma electoral de cara a mejorar nuestras instituciones democráticas.

\section{Elementos teóricos para el estudio de los sistemas electorales.}

La selección entre la oferta elecloral (los partidos políticos contendientes) se lleva a cabo en un marco instilucional que es reflejo del sistema político de la 
nación. Existe todo un entramado de normas formales e informales que regulan los procesos electorales. Los votantes optan por oferlas partidarias dentro de los límites establecidos por el derecho electoral y el resultado se define según la sistemática electoral. Existe pues, una distinción entre derecho y sistema electoral; este último puede definirse, de acuerdo a varios autores (Vanaclocha, 1989:p.274; Nohlen, 1994:p.34; Rae, 1967:p.14) como el conjunto de elementos de la normativa electoral que, con eficacia direcla, conducen o inciden en la traducción o transformación de las preferencias electorales (votos emitidos) en cuolas de poder institucionalizado (escaños o cargos electivos).

\subsection{Elementos constitutivos de la sistemática elecloral.}

Aunque las investigaciones normalmente enfocan uno o algunos, existe un amplio consenso entre los autores en señalar los siguientes elementos constitutivos o configuradores de los sistemas electorales:

- Número de representantes a elegir.

- Distribución de las circunscripciones o distritos electorales.

- Las formas de candidatura.

- Las formas de expresión del volo o procedimientos de volación.

- Las barreras legales.

— La fórmula electoral.

\section{Número de representantes a elegir.}

Bien puede establecerse como un número fijo o de acuerdo a una relación, ya sea entre escaños y electores o escaños y habitantes. Este elemento constituye el tamaño o magnitud de la circunscripción y permite clasificarlas en pequeñas, medianas y grandes de acuerdo a la tabla siguiente (Cfr. Nohlen, 1994:p.53):

\section{Número de escaños por circunscripción}

$$
\begin{aligned}
& 2-5 \\
& 6-10 \\
& 10 \text { y más }
\end{aligned}
$$

$$
\begin{aligned}
& \text { Circunscripciones } \\
& \text { plurinominales. }
\end{aligned}
$$

circunscripción pequeña circunscripción mediana circunscripción grande

\section{Las circunscripciones.}

Son aquellas zonas en las cuales los votos emitidos por los electores constiluyen el fundamento primero y último para el reparto de los escaños con independencia de los votos que hayan sido otorgados en otras zonas. Normalmente 
las circunscripciones corresponden a divisiones administrativo-lerriloriales aunque no necesariamente ocurre así. De hecho, la manipulación de la distribución de circunscripciones conocida como Gerrymandering utiliza criterios político-partidistas y no precisamente administrativo/territoriales (Nohlen, 1994:pp.50-2). Por otra parte, las circunscripciones pueden ser uninominales (se dispula un escaño) o plurinominales (se reparten varios escaños).

\section{Formas de candidatura o tipo de listas y procedimiento de votación.}

Entre las primeras tenemos la candidatura individual o personal (llamada también unipersonal) normalmente asociada a circunscripciones uninominales (ya sea en sistemas electorales mayoritarios o proporcionales) y la candidatura de lista, donde se establece la relación de candidalos que se presentan a la elección (se asocia a los sistemas proporcionales pero puede encontrarse también en sistemas mayoritarios).

Las candidaturas de lista pueden ser de tres tipos: lista cerrada y blaqueada (cada partido presenta su lista y el orden de los candidatos es invariable; el elector vota la lista y los escaños se atribuyen en el orden en que están fijados en la lista); lista cerrada pero no bloqueada (el elector puede ordenar la lista aunque no pueda agregar candidatos) y la lista abierta (se puede modificar el orden, combinar candidatos de diferenles partidos y hasta agregar nuevos candidatos).

En cuanto al procedimiento de votación se habla de voto único cuando el elector emite un sólo voto independientemente de los candidatos a elegir en la circunscripción. En cambio, cuando el elector puede emitir su volo a favor de varias candidaturas se trata de un voto múltiple. Cuando, ante una lista cerrada y no bloqueada, el elector altera el orden de las candidaturas estamos hablando de un voto preferencial. Y cuando el elector puede incluso combinar candidatos de diferentes listas (abiertas) se trata del llamado voto combinado o "panachage".

Entre formas de candidatura y procedimientos de volación podemos establecer las siguientes relaciones:

- lista cerrada y bloqueada $\longrightarrow$ voto único.

- lista cerrada y no bloqueada $\longrightarrow$ voto preferencial.

- lista abierta $\longrightarrow$ voto combinado o "panachage"

- candidatura unipersonal $\longrightarrow$ voto único.

\section{Barreras legales.}

Para que un partido político cualquiera pueda oblener escaños parlamentarios, e incluso mantener su misma existencia como partido legalizado, se le 
exige, mediante disposiciones especiales, que alcance un umbral mínimo de votos, por lo que se dice que las barreras tienen efectos reductores haciendo que el número de partidos parlamentarios sea menor que el número de partidos electorales. Además, constituyen un mecanismo para evitar la atomización del sistema partidario. Sin embargo, tienen la desventaja que aún en sistemas electorales proporcionales, los votos por los partidos que no alcanzan el umbral mínimo se pierden, son volos inúliles.

\section{La fórmula electoral.}

Se trata del procedimiento matemático para convertir los votos en escaños. Podemos distinguir fórmulas mayoritarias y fórmulas proporcionales. Entre las primeras están: la fórmula de mayoría simple o relativa, las fórmulas de mayoría absoluta y las fórmulas mayoritarias corregidas por determinadas formas de expresión del voto. Entre las fórmulas proporcionales se encuentran: las fórmulas de cociente electoral común (Hare, Hagenbach-Bischoff, Imperialli y los métodos de reparto de escaños en segunda fase tales como los del Resto Mayor, Resto menor y Media más alta), las fórmulas de divisores comunes (D’hont, Saint-Lagüe, Saint-Lagüe modificado, Danesa) y la fórmula de cociente electoral de lista (Niemeyer) utilizada en el sistema del Bundesłag alemán (Cfr. Vanaclocha, op cit:pp.289-97; Nohlen, op cit:pp.66-76; Taagepera \& Shugart, 1989:pp.29-36).

Una de las cosas que vamos a mostrar en este trabajo es la posibilidad de manipular aspectos "lécnicos" como el tamaño de las circunscripciones y su distribución así como la elección de determinada fórnula electoral para producir un resultado particular, en una especie de "ingeniería electoral", que favorezca a unos partidos y afecle negalivamente a otros. Se trata de una forma institucional de influir sobre la decisión de los electores expresada en su volo y de la cual éstos normalmente no se percatan.

El que se hable de fórmulas mayoritarias y fórmulas proporcionales no debe llevamos a identificar mecánicamente a los sistemas mayoritarios con las primeras y a los sistemas proporcionales con las segundas. En este sentido es que Nohlen ha introducido la distinción entre fórmulas (decisorias) y principios de representación (1993:pp.14-8; 1994:pp.92-4). Mientras que las primeras se refieren a la transformación de votos en escaños ( $y$, por tanto, se plantean al nivel de circunscripción), los segundos se refieren a los objetivos políticos de la representación política ( $y$, por tanto, se plantean al nivel del resultado general de las elecciones a escala nacional). En realidad, son los principios de representación los que orientan la selección de los elementos constitutivos de los sistemas electorales. Así es como se definen los dos tipos básicos de sistemas electorales: de mayoría absoluta o simple ("majority/plurality rule") y el proporcional ("proportional representation", PR). 
De acuerdo a los principios de representación, el objetivo político de los sistemas mayoṛitarios (sobre todo en regímenes parlamentarios) es lograr la mayoría para un partido o alianza/coalición de partidos para favorecer la eficacia gubernativa. En tanto que el objelivo político de los sistemas proporcionales es reflejar en los órganos de gobierno, con la mayor exaclitud posible, las fuerzas sociales y grupos políticos entre la población'.

Esta distinción de fórmulas y principios nos permite visualizar teóricamente por qué sistemas electorales proporcionales pueden producir efectos asociados a los sistemas mayoritarios. Como dice Nohlen (1994:pp.97-8), aunque generalmente en los sistemas electorales empíricos la fómula de decisión y el principio de representación se corresponden, bajo determinadas condiciones, la fórmula de decisión mayoritaria se aproxima a los objetivos de la representación proporcional y el método decisorio proporcional se aproxima a los objetivos de la representación por mayoría.

\subsection{Proporcionalidad y efectos reductores.}

La discusión que sigue se refiere explícitamente a los sistemas electorales de representación proporcional (PR) suponiendo que éstos prelenden repartir escaños de acuerdo al total o a la proporción de votos que obtiene cada partido. En este sentido, se ha detectado que ya sea intencionalmente o no se producen efectos desproporcionales y rara vez se obtiene una correspondencia exacta entre proporción de votos y escaños2.

De los elementos constitutivos de los sislemas electorales, son el tamaño o magnitud de las circunscripciones, el tamaño del parlamento, las barreras legales y la fórmula electoral las que suelen asociarse a los problemas de desproporcionalidad. Se dice, por ejemplo, que a menor tamaño de las circunscripciones, o menor tamaño del parlamento, o mayor sea la barrera electoral, menor es la proporcionalidad (Lijphart, 1994:pp.10-4; Nohlen, 1994:pp.52-61)3.

Más allá de criterios "técnicos", la desproporcionalidad producida por un sistema que paradójicamente busca ser proporcional puede obedecer a razones como: crear mayorías legislativas "artificiales", impedir la fragmentación del parlamento y dinamizar la acción parlamenlaria haciendolo efectivo y eficaz.

Entre los indices elaborados para medir la proporcionalidad de un sistema particular y hacer comparaciones con otros sistemas electorales se encuentra el Indice de Proporcionalidad de Rose 4 calculado mediante la fórmula:

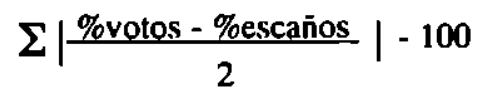


de acuerdo a este índice, entre más cercano esté su valor a 100 mayor será la proporcionalidad del sistema en consideración.

Otro índice utilizado para medir la proporcionalidad, aunque indirectamente a través de la desproporcionalidad, es el índice propuesto por Michael Gallagher ("least-squares index") que se calcula con la fórmula:

$$
\mathrm{LSq}=\sqrt{\left[\frac{1}{2} \Sigma(\mathrm{V} i-\mathrm{Si})^{2}\right]}
$$

donde Vi y Si son las proporciones de votos y escaños para cada partido. Este índice pesa las desviaciones de cada partido por su propio peso, es decir, mayores desviaciones pesan más en el cálculo global que pequeñas desviaciones (cfr. Lijphart, op cit:pp.60-1).

Otra medida útil en el estudio de la proporcionalidad de los sistemas electorales es la desviación electoral que nos indica la sobre o sub-representación de los partidos políticos respecto al nivel de votos. Puede calcularse mediante la fórmula:

$$
\mathrm{DE}=\frac{\% \mathrm{Si} \times 100}{\% \mathrm{Vi}}-100
$$

Valores mayores de este índice nos indican mayor sobre-representación y menores valores indican mayor sub-representación del partido político en cuestión 5 .

Existe otra forma adicional de estudiar la proporcionalidad de un sistema. En la literatura inglesa se conoce como "malapportionment" y se refiere no a la repartición de los escaños entre los partidos sino a la relación electores/escaños que puede violar el principio de igualdad en el valor del volo. Allí donde se disputan menos escaños para una misma cantidad de eleclores, el voto individual tiene un menor valor. En otras palabras, se necesitan más volos para alcanzar un escaño. Por esta vía también se puede favorecer a los partidos más grandes que son los que tienen la posibilidad de alcanzar los votos necesarios para ganar el escaño en dispula y de esla manera también se producen efeclos desproporcionales en el reparto.

En relación a los efectos reductores de los sistemas electorales nos referimos a las posibilidades que tienen los partidos para participar en el reparto de escaños. De hecho la distinción entre partidos electorales y partidos parlamentarios da cuenta de este hecho. Por tanlo, puede establecerse una medida de estos efectos considerando el número de partidos electorales (Nv) y parlamentarios (Ns), asi: 


$$
r=(\mathrm{Nv}-\mathrm{Ns}) / \mathrm{Nv}
$$

el máximo valor para $\mathrm{r}$ es 1 (100\%). A medida que disminuye $\mathrm{r}$ los efectos reductores del sistema son menores hasta el punto en que no hay efectos reductores, es decir, cuando todos los partidos en la contienda electoral han alcanzado al menos un escaño ( $\mathrm{Nv}=\mathrm{Ns}$ ).

Ahora bien, la reducción en Ns puede lograrse vía el establecimiento de barreras legales (ya disculidas anteriormente) o mediante la manipulación del tamaño de la circunscripción o con el uso de determinada fómula electoral que produzcan una especie de barteras teóricas (de inclusión o exclusión) para entrar al reparto de escaños (Taagepera \& Shugart, 1989:pp.274-7; Lijphart, 1994:pp.25-30). Dicho de otra forma, cuando no hay disposiciones legales que establezcan barreras se puede manipular la fórmula y magnilud de las circunscripciones para generar efectos equivalentes a los producidos por las barreras legales; de allí que en estos casos se hable de barreras teóricas.

\section{El sistema electoral salvadoreño para el caso de la Asamblea Legislativa.}

A continuación nos vamos a dedicar al estudio de la proporcionalidad del sistema electoral parlamentario utilizado en El Salvador. Vamos a ver que éste favorece a los tres partidos con mayor fuerza electoral a través de los efectos desproporcionales originados principalmente por la actual distribución (en cuanto a la magnitud) de las circunscripciones. Primero veamos qué establece el Código electoral al respecto.

\subsection{La normatividad del Código Electoral de 1993.}

En primer lugar, sólo pueden volar los mayores de 18 años que inscritos en el Regisıro Electoral poseen su carné electoral. Este es un dalo importante que puede permitir establecer niveles de concurrencia y/o de abslención. Sin embargo, hasta la fecha no se conoce con exactitud la magnitud del Padrón Electoral y ello obliga a trabajar con estimaciones del número de potenciales electores6.

En segundo lugar, para las elecciones de diputados, el Art.12 del Código establece el tamaño y distribución de las circunscripciones electorales para un total de 84 escaños. Fuera de la circunscripción nacional (en que se disputan 20 escaños), el resto de circunscripciones coinciden con la división político-administrativa del país en departamentos. Esto quiere decir que tenemos catorce circunscripciones correspondientes a los 14 departamentos de la repúbli$\mathrm{ca}^{7}$, que según su tamaño pueden clasificarse en: 12 circunscripciones pequeñas, 1 mediana y 2 grandes (cfr. supra). Esta distribución de las circunscripciones en dos niveles (nacional y departamental) hacen que este sistema sea una especie de lo que Lijphart Ilama "Two-fier disfricting system" (1994:p.30). 
Finalmente, el Art.262 del Código Elecloral establece un procedimiento de adjudicación de los escaños a través del cociente electoral simple. Si, luego de esta distribución, existiesen escaños que asignar se ha de seguir la mecónica de residuos o restos mayores. Este procedimiento es válido tanto para las circunscripciones departamentales como para la circunscripción (o "plancha") nacional y la identificaremos como la fórmula Hare-restos mayores.

\section{Cuadro No.1}

Población por departamento y magnitud de las circunscripciones departamentales para las elecciones de 1994.

\begin{tabular}{|c|c|c|c|c|c|c|}
\hline \multirow[b]{2}{*}{ Departamento } & \multicolumn{2}{|c|}{ Población } & \multicolumn{2}{|c|}{ Pob. $\geq 18$ años } & \multicolumn{2}{|c|}{$\mathbf{M}$} \\
\hline & Abs. & $\%$ & Abs. & $\%$ & Abs. & $\%$ \\
\hline 1 Ahuachapán & 261,188 & 5.10 & 130,406 & 4.71 & 3 & 4.69 \\
\hline 2 Santa Ana & 458,587 & 8.96 & 253,714 & 9.17 & 6 & 9.38 \\
\hline 3 Sonsonate & 360,183 & 7.04 & 185,749 & 6.71 & 4 & 6.25 \\
\hline 4 Chalatenango & 177,320 & 3.46 & 86,286 & 3.12 & 3 & 4.69 \\
\hline 5 La Libertad & 513,866 & 10.04 & 277,969 & 10.05 & 5 & 7.80 \\
\hline 6 San Salvador & $1,512,125$ & 29.54 & 893,877 & 32.31 & 16 & 25.00 \\
\hline 7 Cuscatlán & 178,502 & 3.49 & 92,599 & 3.35 & 3 & 4.69 \\
\hline $8 \mathrm{La} \mathrm{Paz}$ & 245,915 & 4.80 & 120,306 & 4.35 & 3 & 4.69 \\
\hline 9 Cabañas & 138,426 & 2.71 & 72,049 & 2.61 & 3 & 4.69 \\
\hline 10 San Vicente & 143,003 & 2.79 & 71,977 & 2.61 & 3 & 4.69 \\
\hline 11 Usulután & 310,362 & 6.06 & 162,682 & 5.88 & 4 & 6.25 \\
\hline 12 San Miguel & 403,411 & 7.88 & 213,056 & 7.70 & 5 & 7.80 \\
\hline 13 Morazán & 160,146 & 3.13 & 77,966 & 2.82 & 3 & 4.69 \\
\hline 14 La Unión & 255,565 & 5.00 & 127,523 & 4.61 & 3 & 4.69 \\
\hline Total & $5,118,599$ & 100 & $2,766,159$ & 100 & 64 & 100 \\
\hline
\end{tabular}

M = Magnitud de la circunscripción. Fuente: DGEC, 1992.

El estudio de la información mostrada en el Cuadro No.1 nos arroja los primeros indicios de problemas en la proporcionalidad lograda a través del sislema electoral vía distribución y magnitud de las circunscripciones. Si bien es cierto que los dalos de población en este cuadro se refieren a 1992, constituyen la base más confiable para nuestros cálculos. De lodas maneras, el Tribunal Supremo Electoral estimó en 2,700,000 los potenciales votantes para 1994 y a los cuales se pretendió registrar y cametizar. Este dato está en el orden de los 2,766,159 mayores de 18 años según el Censo de Población de 1992 publicado 
en 1995. Se sabe que el TSE habría conlado con una muestra censal para hacer sus estimaciones de allí la cercanía en los tolales\$.

Con un total de 1,345,277 votos válidos se concluye que los 84 diputados que forman la Asamblea Legislativa fueron electos por aproximadamente el $48 \%$ de los ciudadanos que polencialmente tenían derecho a voto. Un dato un tanto superior al $40.7 \%$ correspondiente a las elecciones parlamentarias de 1991 si se considera la excepcionalidad de las elecciones de 1994. Como resultado de esta concurrencia estamos ante una Asamblea electa por el $26.3 \%$ de la población total salvadoreña cuando podría haberlo sido (en el mejor de los casos) por el $53.3 \%$.

Al observar la proporcionalidad de escaños asignada a cada circunscripción departamental salta a la vista que La Libertad, pese a tener una mayor proporción de habitantes y ciudadanos tiene asignada una menor proporción parlamentaria comparada con Santa Ana. Por otra parte, Ahuachapán tiene casi el doble de la población, total y mayor de $\mathbf{1 8}$ años, que Cabañas y San Vicente. Sin embargo, tiene asignados igual cantidad de escaños. Finalmente, Chalatenango, Cabañas, San Vicente y Morazán tienden a estar sobre-representados por casi dos puntos porcentuales en tanto San Salvador estaría sub-representado por 7 puntos porcentuales. Si estas observaciones nos parecen indicar que no hay una distribución proporcional de los escaños de acuerdo a la distribución territorial de la población vale la pena preguntarse ¿cuál ha sido entonces el criterio para asignar los escaños a cada circunscripción? Al respecto hay que decir que el actual Código Electoral no establece tal criterio y, por tanto, no está claro a qué obedece esa distribución.

\subsection{Desigualdad en el valor del voto.}

El cuadro No. 2 nos da clara información sobre la mala distribución de diputados ("malapportionment") y el cociente electoral de tal manera que nos es posible postular no sólo la desigualdad en el valor del voto sino también en el valor del ciudadano mismo. Para que esto no ocurriese no sólo sería necesario establecer que por tantos volos válidos se disputaría un escaño sino también que por tantos ciudadanos habría un diputado que los representa en el parlamento.

En relación al "malapportionment" es evidente como cada ciudadano de Cabañas, San Vicente y Morazán vale por dos de La Libertad y San Salvador. También se observa como aunque la media a nivel nacional ande en los 46,000 ciudadanos por diputado, a excepción de Sonsonate, todos los departamentos muestran gran dispersión estándo los extremos (San Salvador y San Vicente) cerca de los 10,000 y 20,000 ciudadanos por diputado arriba o abajo de la media. 
Cuadro No.2

"Malapportionment" y Cociente Elecloral por circunscripción en 1994.

\begin{tabular}{|c|c|c|c|c|c|c|c|c|}
\hline Circunscripción & Pobz 18x/\% & V.Válidas & M & $M(\%$ & Pob_IBa/M & Malapp." & CocElec. & SR \\
\hline Ahuachapán & 4.71 & 60,388 & 3 & 4.69 & 43,468 & 1.00 & 20,129 & -0.02 \\
\hline Santa Ana & 9.17 & 133,667 & 6 & 9.38 & 42,285 & 0.98 & 22,277 & +0.21 \\
\hline Sonsonate & 6.71 & 106,043 & 4 & 6.25 & 46,437 & 1.07 & 26,510 & -0.46 \\
\hline Chalatenango & 3.12 & 50,252 & 3 & 4.69 & 28,762 & 0.67 & 16,750 & +1.57 \\
\hline La Libertad & 10.05 & 151,816 & 5 & 7.80 & 55,593 & 1.29 & 30,363 & -2.25 \\
\hline San Salvador & 32.31 & 421,206 & 16 & 25.00 & 55,867 & 1.29 & 26,325 & -7.31 \\
\hline Cuscatlán & 3.35 & 48,602 & 3 & 4.69 & 30,866 & 0.71 & 16,200 & +1.34 \\
\hline La Paz & 4.35 & 68,329 & 3 & 4.69 & 40,102 & 0.93 & 22,776 & +0.34 \\
\hline Cabañas & 2.61 & 30,375 & 3 & 4.69 & 24,016 & 0.56 & 10,125 & +2.08 \\
\hline San Vicente & 2.61 & 37,426 & 3 & 4.69 & 23,992 & 0.56 & 12,475 & +2.08 \\
\hline Usulután & 5.88 & 71,787 & 4 & 6.25 & 40,670 & 0.94 & 17,946 & +0.37 \\
\hline San Miguel & 7.70 & 83,605 & 5 & 7.80 & 42,611 & 0.99 & 16,721 & +0.10 \\
\hline Morazán & 2.82 & 35,812 & 3 & 4.69 & 25,988 & 0.60 & 11,937 & +1.87 \\
\hline La Unión & 4.61 & 45,969 & 3 & 4.69 & 42,507 & 0.98 & 15,323 & +0.08 \\
\hline
\end{tabular}

- Malapportionment $=$ Pob $\geq 18 \mathrm{años}(\%) / \mathrm{M}(\%)$.

SR = Sobre o sub-representación, \%M - \%P.

Fuenles: DGEC, 1992;

TSE, 1994a.

y cálculos propios.

El efecto de esta particular distribución de las circunscripciones vuelve a hacerse evidente al considerar el cociente elecloral necesario en cada circunscripción para agenciarse un escaño parlamentario. La circunscripción más "cara" resulta ser La Libertad en tanto que la más "barata" es Cabañas. También es curioso que La Libertad y San Miguel tengan asignados igual número de escaños pero que se necesite casi el doble de votos por diputado en la primera frente a los necesarios en el segundo. De manera semejante, si comparamos Ahuachapán, La Paz y Cabañas se hace visible de nuevo la desigualdad en el valor del voto puesto que contando las tres con el mismo número de escaños (tres) es necesario el doble de votos válidos en las primeras dos en relación a Cabañas para distribuir dichos escaños.

Por otra parte, Sonsonate tuvo más votos válidos que San Miguel, y La Libertad más votos válidos que Santa Ana. Sin embargo, tanto Sonsonate como La Libertad tienen un escaño menos que San Miguel y Santa Ana, respectivamente. Así las cosas, a pesar de tener más volos válidos hay menos escaños que repartir en Sonsonate y La Liberlad9. Podrían hacerse más comparaciones, sin 
embargo creemos que las aquí señaladas justifican la pregunta: ¿a quién favorece esta situación? La teoría establece que circunscripciones pequeñas favorecen a los partidos grandes en tanto que circunscripciones grandes favorecen a los partidos pequeños.

\subsection{Efectos desproporcionales y reductores del sistema electoral.}

Vamos a utilizar la información del cuadro No.3 para estudiar más de cerca la proporcionalidad del sistema electoral salvadoreño. El cuadro sólo incluye a los partidos que sobrepasaron la barrera legal que el código electoral establece en el $1 \%$, de allí que los porcentajes tolales de votos no sumen el $100 \%$.

En primer lugar, podemos apreciar que en general ARENA, PDC, PCN y el FMLN obtuvieron una proporción de escaños mayor que su proporción de votos. Las excepciones son 1985 para ARENA y 1994 para el PCN. El resto de partidos (PPS, AD, PAISA, CD, MAC, UDN y MU) obluvieron siempre una proporción de escaños menor que su proporción de volos.

Si traemos a cuenta que en 1985, ARENA y el PCN fueron en coalición a las elecciones pero manteniendo sus emblemas separados en la papeleta de votación, podemos decir que la distribución de escaños entre estos partidos fue producto de la política de alianzas más que del sistema electoral. Si, por otra parte, tomamos en cuenta que el PCN ocupó el tercer lugar en 1982, 1985 y 1988, y pasó a la cuarta posición en 1994, por debajo de ARENA, FMLN y PDC, entonces podríamos aventurarnos a decir que el sistema electoral favorece a los tres partidos más fuertes electoralmente hablando. El hecho que $C D$ no se haya visto favorecida por esta tendencia en 1991 y sí el PCN, a pesar de ocupar el cuarto lugar, puede explicarse por la mayor experiencia electoral de este último y por la introducción de la "plancha" nacional ese año.

En segundo lugar, con la información del Cuadro No.3 hemos calculado los índices de Rose y Gallagher ( $\mathrm{LSq}$ ), mostrados en el Cuadro No.4, que son otra forma de aproximamos a la proporcionalidad y a los efectos desproporcionales del sistema electoral 10. También hemos agregado el índice " $r$ " para estudiar los efectos reductores del sistema.

Como puede notarse, 1985 es el año que mayor desproporción presenta. Ya mencionamos que el reparto de escaños negociado entre ARENA y el PCN está a la base de este efecto. Por otra parte, 1988 fue el año en que sólo ARENA, PDC y PCN alcanzaron escaños quedándose 5 partidos fuera del reparto (2 por no alcanzar el $1 \%$ de volos como barrera legal para no ser cancelado como partido político y $\mathbf{3}$ por no alcanzar una barrera teórica o efectiva aproximadamente del $4.5 \%$ de los votos válidos) ${ }^{\prime}$. 


\section{Cuadro No.3}

Votos y Escaños alcanzados por los partidos contendientes en las elecciones parlamentarias del período 1982-1994.

\begin{tabular}{|c|c|c|c|c|c|c|c|c|c|c|}
\hline \multirow[t]{2}{*}{ Partido } & \multicolumn{2}{|c|}{1982} & \multicolumn{2}{|c|}{1985} & \multicolumn{2}{|c|}{1988} & \multicolumn{2}{|c|}{1991} & \multicolumn{2}{|c|}{1994} \\
\hline & $\% \mathrm{~V}$ & $\% \mathrm{~S}$ & $\% \mathrm{~V}$ & $\% \mathrm{~S}$ & $\% V$ & $\% s$ & $\% \mathrm{~V}$ & $\% S$ & $\% \mathrm{~V}$ & $\% \mathrm{~S}$ \\
\hline ARENA & 29.5 & 31.7 & 29.7 & 21.6 & 48.0 & 50.0 & 44.3 & 46.4 & 45.0 & 46.4 \\
\hline PDC & 40.1 & 40.0 & 52.3 & 55.0 & 35.2 & 38.3 & 28.0 & 31.0 & 17.9 & 21.4 \\
\hline PCN & 19.2 & 23.3 & 8.4 & 20.0 & 8.6 & 11.7 & 9.0 & 10.7 & 6.2 & 4.8 \\
\hline PPS & 2.9 & 1.7 & 1.7 & 0.0 & & & & & & \\
\hline$A D$ & 7.4 & 3.3 & 3.7 & 1.7 & 1.7 & 0.0 & & & & \\
\hline PAISA & & & 3.7 & 1.7 & 2.1 & 0.0 & & & & \\
\hline LIBERACION & & & & & 3.7 & 0.0 & & & & \\
\hline $\mathrm{CD}$ & & & & & & & 12.1 & 9.5 & 4.4 & 1.2 \\
\hline MAC & & & & & & & 3.2 & 1.2 & & \\
\hline UDN & & & & & & & 2.7 & 1.2 & & \\
\hline FMLN & & & & & & & & & 21.4 & 25.0 \\
\hline $\mathbf{M U}$ & & & & & & & & & 2.5 & 1.2 \\
\hline Total & 99.1 & 100.0 & 99.5 & 100.0 & 99.38 & 100.0 & 99.3 & 100.0 & 97.4 & 100.0 \\
\hline
\end{tabular}

Fuente: CCE 1982a, 1985a, 1988a, 1991 a.

TSE 1994a.

\section{Cuadro No.4}

Proporcionalidad y Efectos reductores del sistema electoral salvadoreño en el caso de las elecciones parlamentarias, 1982-1994.

\begin{tabular}{lcccccc}
\hline Indice & 1982 & 1985 & 1988 & 1991 & 1994 \\
\hline I.de Rose & & 94.15 & 85.95 & 92.15 & 93.55 & 92.80 \\
Gallagher & 4.47 & 10.45 & 4.70 & 3.83 & 4.53 \\
\#Pelim.xBL & 1. & 3. & 2. & 1. & 3. \\
\#Pelim.xBT & 0. & 1. & 3. & 0. & 0. & \\
$\mathrm{r}^{*}$ & 0.17 & 0.44 & 0.63 & 0.14 & 0.33 \\
$\mathrm{r}^{* *}$ & & 0.00 & 0.17 & 0.50 & 0.00 & 0.00 \\
\hline
\end{tabular}

\#Pelim.xBL = \# de partidos eliminados por barrera legal.

\#Pelim.xBT = \# de partidos eleminados del reparto de escaños por no alcanzar la barrera teórica.

* = considerando todos los partidos.

** = sin considerar los partidos excluidos por bartera legal.

Nota: Para I. de Rose y Gallagher, los cálculos basados en los partidos que superaron la barrera legal. 
Es oportuno en estos momentos agregar unas palabras sobre los efectos reductores del sistema. Como se ve en este último cuadro, en todas las elecciones ha sido eliminado por lo menos un partido político. Esto hace que, como efecto mecánico del sistema electoral, no se incremente el formato del sistema de partidos y, como efecto psicológico, sean casi siempre los mismos partidos los que controlan más del $90 \%$ de los escaños. De esta manera, los efeclos reductores operarían en favor de los Ires primeros partidos dado que la dispersión de votos en partidos pequeños no representaría amenaza al potencial parlamentario de aquéllos 12 .

Combinando los cuadros Nos. 3 y 4 podemos concluir, empíricamente hablando, que los partidos pequeños y los nuevos partidos deben superar tres barreras impuestas por el sistema electoral para no verse en desventaja frente a los partidos mayores:

a) la barrera legal del $1 \%$ de los votos válidos que establece el Código Electoral para mantener su existencia.

b) la barrera teórica o umbral de representación (Nohlen, 1994:p.54), cuando existe, para entrar al reparto de escaños.

c) la barrera que es necesario superar para convertirse en la tercera fuerza electoral y que permitiría alcanzar una proporción de escaños mayor que la proporción de votos. Esta barrera coincidiría con el llamado "Break-even point" que, según la fórmula propuesta por Taagepera \& Shugart (1989:p.88), habría tenido un valor de $17.7 \%$ en 1994 .

\section{Cuadro No.5}

Desviación Electoral para los partidos parlamentarios entre 1982-1994.

\begin{tabular}{lrrrrr}
\hline Partido & 1982 & 1985 & 1988 & 1991 & 1994 \\
\hline ARENA & 7.46 & -27.27 & 4.17 & 4.74 & 3.11 \\
PDC & -0.24 & 5.16 & 8.81 & 10.71 & 19.55 \\
PCN & 21.35 & 138.10 & 36.05 & 18.89 & -22.58 \\
PPS & -41.38 & & & & \\
AD & -55.41 & -54.05 & & & \\
PAISA & & -54.05 & & & \\
CD & & & & -21.49 & -72.73 \\
MAC & & & & -52.50 & \\
UDN & & & & & 16.86 \\
FMLN & & & & & -52.00 \\
MU & & & & & \\
\hline
\end{tabular}

Nota: Elaborado con base en la información del Cuadro No.3 y la fórmula $\mathrm{DE}=(\% \mathrm{Si} \times 100) / \% \mathrm{Vi}-100$, donde $\mathrm{Si}$ y $\mathrm{Vi}$ son escaños y volos del i-ésimo partido respectivamente. 
Adicionalmente, el cuadro No.5 es otra forma de argumentar la ventaja que el sistema electoral proporciona a los tres principales partidos. Este cuadro nos sugiere que quien históricamente se ha visto más favorecido ha sido el partido que electoralmente ha constiluido la tercera fuerza: el PCN entre 1982 y 1991, y el PDC en 1994.

\section{EJ sistema electoral para los Concejos Municipales.}

En primer lugar se trata de un sistema de mayoría simple (plurality en inglés). Por tanto, no importa cuál sea la diferencia entre el partido político con más volos y el resto de partidos. Un voto puede ser la diferencia y el concejo municipal lo ganará el partido con el mayor número de votos válidos. Ahora bien, de acuerdo al artículo 13 del Código Electoral: "En cada Municipio, se elegirá un Concejo Municipal compuesto por un Alcalde, un Síndico, dos Regidores y cuatro Miembros Suplentes, para sustituir indistintamente a cualquier propietario...". Es decir que la elección no es unicamente del Alcalde, como populamente se plantea en una especie de "presidencialismo municipal", sino que se elige un grupo de ocho ciudadanos como mínimo para el gobiemo local. Esto quiere decir que por cada municipio en que un partido obtiene la mayoría de votos logra conformar un gobiemo municipal monocolor de por lo menos ocho representantes.

Dicho de otra forma, el partido ganador en la elección municipal no sólo gana la Alcaldia (el puesto de Alcalde) sino que, sin importar la diferencia de votos, gana también la conformación de todo el concejo municipal y, por tanto, se trata de una lista de candidatos ganadora en circunscripciones plurinominales.

Decimos que el partido ganador obtiene como mínimo ocho representantes en el gobiemo municipal porque en el mismo artículo 13 se señala el número de Regidores que es necesario adicionar en aquellas poblaciones de más de cinco mil habitantes. El cuadro No.6 resume el tamaño del Concejo Municipal según la población del municipio respectivo de acuerdo a lo establecido en el articulo mencionado.

Cuadro No.6

Concejales según población municipal

Población del municipio \# de Concejales

$\begin{array}{lr}\text { < de } 5000 \text { hab. } & 8 \\ 5000-10,000 \text { hab. } & 10 \\ 10.000-20,000 \text { hab. } & 12 \\ 20,000-50,000 \text { hab. } & 14 \\ 50,000-100,000 \text { hab. } & 16 \\ >\text { de } 100,000 \text { hab. } & 18\end{array}$


Como puede inferirse, con simple mayoría de votos un partido puede llegar a obtener hasta los dieciocho representantes del gobierno de un municipio de más de cien mil habitantes como es el caso de San Salvador. Ahora bien, el que la competencia por una lista cerrada y bloqueada se lleve a cabo en circunscripciones plurinominales y se decida por simple mayoría no sólo produce efectos desproporcionales como es lógico. Puede llegar a favorecer la consolidación del poder de un partido en el nivel municipal. Si esto se repite en muchos municipios puede entonces favorecer la consolidación de dicho partido a nivel departamental $\mathrm{e}$ incluso nacional 13 .

Vamos a examinar los resultados electorales municipales de 1994 para ver en cuántos de los 262 municipios en disputa, el partido ganador no obtuvo mayoría absoluta y por simple mayoría logró que los votos contrarios no tuviesen ninguna eficacia en la conformación de la representación política municipal. Es decir, vamos a ver en cuántos municipios la suma de los partidos perdedores es mayor que el total de votos del partido ganador y, sin embargo dado el lipo de lista, el procedimiento de votación y la fórmula de decisión, ninguno obtuvo representación en el gobierno municipal. Los cuadros No.7(a) y (b) presentan la distribución de alcaldías por partido ganador y el total de municipios en que dicho partido obtuvo mayoría absoluta.

\section{Cuadro No.7(a)}

Alcaldías obtenidas por cada partido o coalición por departamento en las elecciones de 1994.

\begin{tabular}{lrrrrrrrr}
\hline Departamento & ARENA & FMLN & FMLN-CD & MAC & PCN & PDC & Total \\
\hline Ahuachapán & 10 & 0 & 0 & 0 & 0 & 2 & 12 \\
Santa Ana & 10 & 0 & 0 & 0 & 0 & 3 & 13 \\
Sonsonate & 13 & 0 & 0 & 0 & 0 & 3 & 16 \\
Chalatenango & 25 & 4 & 0 & 0 & 1 & 3 & 33 \\
La Libertad & 21 & 0 & 0 & 0 & 0 & 1 & 22 \\
San Salvador & 15 & 2 & 0 & 0 & 2 & 0 & 19 \\
Cuscatlán & 14 & 0 & 1 & 0 & 1 & 0 & 16 \\
La Paz & 18 & 0 & 0 & 0 & 2 & 2 & 22 \\
Cabañas & 7 & 1 & 0 & 0 & 1 & 0 & 9 \\
San Vicente & 8 & 1 & 1 & 0 & 0 & 3 & 13 \\
Usulután & 18 & 0 & 0 & 0 & 1 & 4 & 23 \\
San Miguel & 16 & 0 & 0 & 1 & 0 & 3 & 20 \\
Morazán & 17 & 5 & 0 & 0 & 1 & 3 & 26 \\
La Unión & 15 & 0 & 0 & 0 & 1 & 2 & 18 \\
\hline Totales & 207 & 13 & 2 & 1 & 10 & 29 & 262 \\
\hline
\end{tabular}

Fuente: TSE, 1994b. 
Cuadro No.7(b)

Total de municipios por departamento ganados por mayoría absoluta por partido en 1994.

\begin{tabular}{llr}
\hline Departamento & Partido & \#Municipios \\
\hline Ahuachapán & ARENA & 1 \\
Santa Ana & ARENA & 3 \\
& PDC & 1 \\
Sonsonate & ARENA & 4 \\
Chalatenango & ARENA & 11 \\
& FMLN & 4 \\
La Libertad & ARENA & 13 \\
San Salvador & ARENA & 2 \\
Cuscatlán & ARENA & 11 \\
& FMLN-CD & 1 \\
& PCN & 1 \\
La Paz & ARENA & 9 \\
Cabañas & ARENA & 3 \\
& FMLN & 1 \\
San Vicente & ARENA & 2 \\
& PDC & 2 \\
Usulután & ARENA & 4 \\
& PDC & 1 \\
San Miguel & ARENA & 5 \\
Morazán & PDC & 2 \\
La Unión & ARENA & 5 \\
\hline & FMLN & 1 \\
\hline
\end{tabular}

Nota: elaborado en base a TSE, 1994b.

De acuerdo a éstos y al cuadro No.8 que resume esta información, de los 262 municipios en dispula sólo 95 fueron ganados por mayoría absoluta independientemente del partido político vencedor. El resto, es decir, 167 municipios fueron ganados por mayoría simple, lo cual significa que en estos 167 municipios los partidos perdedores tienen, en conjunto, más votos válidos que el partido ganador y sin embargo ninguno logró algún concejal. En estas condiciones se favorece el monopolio de un solo partido de los puestos de auloridad a nivel del municipio. En la medida en que estos municipios son importantes, como las cabeceras departamentales, se favorece el predominio político de un solo partido en el nivel departamental. Es interesante notar que de las catorce cabeceras 
departamentales, únicamente Cojutepeque (correspondiente al departamento de Cuscatlán) fue ganada por mayoría absoluta.

\section{Cuadro No.8}

Distribución de los municipios según el tipo de mayoría alcanzada por el partido ganador.

\begin{tabular}{lrrr}
\hline DEPARTAMENTO & (a) & (b) & Total \\
\hline Ahuachapán & 11 & 1 & 12 \\
Santa Ana & 9 & 4 & 13 \\
Sonsonate & 12 & 4 & 16 \\
Chalatenango & 18 & 15 & 33 \\
La Libertad & 9 & 13 & 22 \\
San Salvador & 17 & 2 & 19 \\
Cuscallán & 3 & 13 & 16 \\
La Paz & 13 & 9 & 22 \\
Cabañas & 5 & 4 & 9 \\
San Vicente & 9 & 4 & 13 \\
Usulután & 18 & 5 & 23 \\
San Miguel & 13 & 7 & 20 \\
Morazán & 20 & 6 & 26 \\
La Unión & 10 & 8 & 18 \\
\hline Tolal & 167 & 95 & 262 \\
\hline
\end{tabular}

(a) \# de municipios en que el partido ganador es minoría.

(b) \# de municipios en que el partido ganador tiene mayoría absoluta.

Como decíamos más arriba, esta situación puede favorecer la consolidación de un mismo partido o coalición en el nivel nacional por la simple agregación de municipios en donde es primera fuerza electoral. Como ilustración, el cuadro No.9 nos muestra, en este sentido, la evolución de la fuerza política de ARENA a nivel de Concejos Municipales.

Según puede notarse, hay una tendencia ascendente en el número de municipios que desde 1985 controla el partido ARENA. No se tomaron en cuenta los datos de 1982 en tanto que el reparto de alcaldías se llevó a cabo no por elección directa sino en base a negociaciones entre los principales partidos que compitieron en la elección de Asamblea Constituyente en ese año. Por otro lado, como veremos posteriormente, exceptuando el caso de Cabañas, el incremento generalizado en el número de municipios que controla ARENA se da a partir de 
1988, año en el que habría ocurrido una elección crítica y un realineamiento electoral14.

\section{Cuadro No.9}

Tolal de Alcaldías ganadas por el partido ARENA desde 1985 hasta 1994 (cifras absolutas y porcentuales).

\begin{tabular}{lrrrrrrr}
\hline Departamento & $1985^{*}$ & \multicolumn{1}{c}{1988} & \multicolumn{1}{c}{1991} & \multicolumn{1}{c}{1994} & Munic. \\
\hline Ahuachapán & $5(41.2)$ & $8(66.7)$ & $8(66.7)$ & 10 & $(83.3)$ & 12 \\
Santa Ana & $5(38.5)$ & $10(76.9)$ & $12(92.3)$ & 10 & $(76.9)$ & 13 \\
Sonsonate & $8(50.0)$ & $11(68.9)$ & $10(62.5)$ & 13 & $(81.3)$ & 16 \\
Chalatenango & $5(50.0)$ & $18(54.5)$ & $16(48.5)$ & 25 & $(75.8)$ & 33 \\
La Libertad & $11(50.0)$ & $19(86.4)$ & $20(90.9)$ & 21 & $(95.5)$ & 22 \\
San Salvador & $3(15.8)$ & $15(78.9)$ & $15(78.9)$ & 15 & $(78.9)$ & 19 \\
Cuscatlán & $13(81.3)$ & $16(100.0)$ & $15(93.4)$ & 14 & $(87.5)$ & 16 \\
La Paz & $15(68.2)$ & $19(86.4)$ & $19(86.4)$ & 18 & $(81.8)$ & 22 \\
Cabañas & $7(77.8)$ & $7(77.8)$ & $8(88.9)$ & 7 & $(77.8)$ & 9 \\
San Vicente & $3(23.1)$ & $4(30.8)$ & $7(53.8)$ & 8 & $(61.5)$ & 13 \\
Usulután & $6(26.1)$ & $16(69.6)$ & $14(60.9)$ & 18 & $(78.3)$ & 23 \\
San Miguel & $7(35.0)$ & $13(65.0)$ & $9(45.0)$ & 16 & $(80.0)$ & 20 \\
Morazán & $8(30.8)$ & $13(50.0)$ & $6(23.1)$ & 17 & $(65.4)$ & 26 \\
La Unión & $3(16.7)$ & $9(50.0)$ & $14(77.8)$ & 15 & $(83.3)$ & 18 \\
\hline
\end{tabular}

"En coalición con el PCN.

- Para un total de 13 municipios; no se cuenta con datos para 18 municipios de este deparamento.

Fuente: CCE 1985b, 1988b, $1991 \mathrm{~b}$.

TSE 1994c.

Finalmente, el cuadro No.10 establece la comparación entre proporción de municipios que controla ARENA y su proporción de votos. Claramente se observa que a pesar de no oblener incrementos sustanciales en esta última (salvo en 1988), la proporción de alcaldías que controla tiende a aumentar. ¿No es acaso ésto un resultado del sistema electoral y de sus efectos sobre el sistema de partidos? 
Cuadro No.10

Evolución del control de municipios y del total de votos obtenidos por ARENA según departamento (porcentajes).

\begin{tabular}{lrrrrrrrr}
\hline \multirow{2}{*}{ Departamento } & \multicolumn{2}{c}{1985} & \multicolumn{2}{c}{1988} & \multicolumn{2}{c}{1991} & \multicolumn{2}{c}{1994} \\
& \%mun & \%vol & \%mun & \%vot & \%mun & \%vot & \%mun & \%vol \\
\hline Ahuachapán & 41.2 & 46.7 & 66.7 & 43.2 & 66.7 & 37.2 & 83.3 & 40.9 \\
Santa Ana & 38.5 & 39.2 & 76.9 & 43.9 & 92.3 & 44.4 & 76.9 & 44.1 \\
Sonsonate & 50.0 & 42.3 & 68.9 & 45.8 & 62.5 & 40.1 & 81.3 & 42.8 \\
Chalatenango & 15.2 & 39.3 & 54.5 & 39.1 & 48.5 & 42.0 & 75.8 & 44.8 \\
La Libertad & 50.0 & 39.1 & 86.4 & 48.7 & 90.9 & 48.2 & 95.5 & 48.0 \\
San Salvador & 15.8 & $34.8 *$ & 78.9 & 51.5 & 78.9 & 49.1 & 78.9 & 43.9 \\
Cuscallán & 81.3 & 51.9 & 100.0 & 58.7 & 93.4 & 53.2 & 87.5 & 51.9 \\
La Paz & 68.2 & 42.9 & 86.4 & 47.6 & 86.4 & 47.1 & 81.8 & 44.5 \\
Cabañas & 77.8 & 52.1 & 77.8 & 52.4 & 88.9 & 46.5 & 77.8 & 49.9 \\
San Vicente & 23.1 & 40.1 & 30.8 & 46.6 & 53.8 & 46.2 & 61.5 & 42.3 \\
Usulután & 26.1 & 36.2 & 69.6 & 54.2 & 60.9 & 42.1 & 78.3 & 43.5 \\
San Miguel & 35.0 & 35.0 & 65.0 & 44.6 & 45.0 & 42.1 & 80.0 & 43.6 \\
Morazán & 30.8 & 38.5 & 50.0 & 40.2 & 23.1 & 34.2 & 65.4 & 39.1 \\
La Unión & 16.7 & 34.2 & 50.0 & 45.5 & 77.8 & 40.1 & 83.3 & 45.1 \\
\hline Nacional & 37.8 & 39.0 & 67.9 & 48.0 & 66.0 & 45.3 & 79.0 & 44.5 \\
\hline
\end{tabular}

- Coalición ARENA-PCN.

- Coalición ARENA-PCN-PAISA para el municipio de San Salvador + Coalición ARENA-PCN para los restantes 18 municipios de este departamento.

\section{Buscando la clave}

Hemos examinado en este trabajo los efectos desproporcionales y reductores del sistema electoral salvadoreño, en especial para el caso de las elecciones legislativas. Hemos visto cómo son los partidos grandes los que se ven favorecidos por estos efectos.

En cuanto a la reducción en el número de partidos, hemos constatado la existencia no sólo de barreras legales sino también hipotéticas o teóricas que operan para evitar el incremento en el formalo o la atomización del sisterna de partidos así como también operan para reducir el reparto de los escaños legislativos favoreciendo así a los partidos grandes que son los que tienen mayores posibilidades para ganarlos.

En cuanto a los efectos desproporcionales del sistema hemos visto cómo ha sido el comportamiento de diferentes índices en el período 1982-1994. Hemos 
establecido que dichos efectos obedecen, entre otras variables, a la fórmula electoral que se aplica y a la magnitud y distribución de las circunscripciones. Aquí puede residir "la clave" para sacar ventaja en elecciones parlamentarias (Artiga-González, 1993). El cuadro No.11 muestra una simulación de lo que habría sido el reparto de escaños en 1994 al variar la lórmula y las circunscripciones 15 . Al calcular el índice de proporcionalidad de Rose para cada columna del cuadro obtenemos los valores $92.8,98.7,86.8$ y 97.5 .

Si mantenemos la fórmula pero variamos la distribución y magnitud de las circunscripciones se eleva sustancialmente el índice de Rose. Si mantenemos la distribución de circunscripciones y variamos la fórmula se produce un descenso sustancial en la proporcionalidad. Si los escaños se disputaran en una sola circunscripción nacional, y sólo variara la fórmula, el índice de proporcionalidad sería muy cercano al oblenido en el primer caso aunque superior al que el actual sistema alcanza.

\section{Cuadro No.11}

Distribución de escaños legislativos por partido según diferentes fórmulas y circunscripciones electorales en 1994.

\begin{tabular}{lccccc}
\hline PARTIDO & Oficial & a. & b. & c. & \%Votos \\
\hline ARENA & 38 & 38 & 47 & 40 & 45.03 \\
PDC & 18 & 15 & 18 & 15 & 17.87 \\
PCN & 4 & 6 & 1 & 5 & 6.21 \\
FMLN & 21 & 18 & 16 & 19 & 21.39 \\
CD & 1 & 4 & 2 & 3 & 4.45 \\
MU & 1 & 3 & 0 & 2 & 2.49 \\
\hline TOTAL & 84 & 84 & 84 & 84 & 97.44 \\
Ind. de Rose & 92.8 & 98.7 & 86.8 & 97.5 & \\
\hline
\end{tabular}

a. Considerando una sola circunscripción nacional manteniendo

b. Considerando fórmula D'Hondt pero manteniendo distribución actual de circunscripciones.

c. Considerando fórmula D'Hondı en una sola circunscripción nacional.

Si la proporcionalidad que se obtiene utilizando Hare-reslos mayores es muy cercana a la obtenida con D'Hondt, considerando en ambas una sola circunscripción nacional, podemos decir que "la clave" no está en la fórmula sino en la magnitud y dislribución de las circunscripciones. Este hecho ya lo anunciaba la teoría (Cfr. Taagepera \& Shugart,1989:pp.112-125). Todavía más, el análisis 
realizado sobre el malapportionment nos reveló que la actual distribución de las circunscripciones no sólo produce efectos desproporcionales sino también atenta contra el principio democrálico de "una persona, un voto". Es decir, produce una desigualdad efectiva en el valor del volo de los ciudadanos según sea la circunscripción donde residen. De paso, esta siluación puede ser manejada por los partidos para buscar alcanzar más escaños con menos votos.

Finalmente, vimos cómo el sistema electoral utilizado para la elección de Concejos Municipales le ha venido permitiendo al partido ARENA agenciarse cada vez más alcaldías sin que haya tenido un sustancial incremento en su fuerza electoral desde 1988. Los datos revelan (cuadro No.10) que incluso con un leve descenso en su proporción de votos entre 1991 y 1994, ARENA obtuvo un incremento de 13 puntos porcentuales en el control de municipios, hecho que puede estar favoreciendo (si no indicando) su consolidación como primer fuerza político-electoral del país.

\section{Notas:}

1. Sartori también sugiere que para hacer la clasificación de los sistemas electorales hay que separar el crilcrio o método de clección, de su resultado, es decir, la forma como se refleja la distribución de volos de los electores (Cfr. Sartori,1994:pp.15-7).

2. Es obvio que los sistemas de represcntación mayoritaria producen efeclos desproporcionales sobre todo cuando la forma de candidatura es plurinominal.

3. Un análisis más detallado sobre los efectos de la fórmula y la magnitud de la circunscripción sobre la proporcionalidad también pucde verse en Lijphart, 1990.

4. Este índice ha sido elaborado como un desarrollo de los índices propuestos por Rae y Loosemore-Hanby, citados en Lijphar, op cit:pp.58-60. Cfr. también la nola 3 al capítulo 3 de la mencionada obra para una breve reseña bibliográfica en tomo a la discusión sobre el índice de proporcionalidad. Vale decir lambién que Taagepera \& Shugart se basan en el índice de Loosemore-Hanby en su Irabajo, cfr. 1989:pp.104-11.

5. Lijphart utiliza la mayor sobre-representación de uno de los partidos más grandes también como medida de la desproporcionalidad (1994:p.62).

6. Ciertamente desde las elecciones de asamblea constituyente en 1982 no se ha logrado establecer el total de electores dadas las condiciones bélicas que prevalecieron en el país. Esto permitió a algunos hablar de "fraude masivo en el número de volantes, aunque no en la proporción de volos." En más de una clección del periodo 82-94 (Cfr. Edilorial ECA, 1982:p.240-3; CIDAl, 1984:pp.203-206).

7. Se trata de 14 circunscripciones plurinominales distribuidas así: 1 de 16 escaños, 1 de 6,2 de 5,2 de 4 y 8 de 3 . La forma de candidatura es de lista cerrada y bloqueada, y el elector cuenta con un voto único.

8. De hecho, la muestra censal que se dió a conocer en Agosto de 1993, y con la que trabajaría el TSE, estimó en 2,739,473 la población con Cćdula de Identidad Personal y que, por tanlo, sería mayor de 18 años.

9. Esta misma situación ocurrió en las elecciones de 1991 lo cual nos hace pensar que no es una mera casualidad. Por el contrario, asi funciona el sistema electoral y cllo puede significar una "carta escondida" con la cual se puede "manufacturar" una mayoría parlamentaria (Artiga-González, 1993:pp.472-475). 
10. Como dato comparativo podemos señalar que, según el índice de proporcionalidad de Rose, el sistema electoral salvadoreño utilizado para la elección de los diputados de la Asamblea Legislativa da niveles de proporcionalidad muy parecidos a los sistemas de Israel, Porlugal, Bélgica o Noruega (CIr. Nohlen, 1994:p.103).

11. Calculada según la fórmula Teff $=50 /(M+1)+50 / 2 M$ propuesla por Lijphart (1994:p.27) con $M=16$ para San Salvador.

12. Si conjuntamente los tres primeros parlidos se llevan alrededor del $90 \%$ de los escaños, el resto de partidos pelean apenas un 10\% que desde 1991 se reduce, en cifras absolutas, a 8 de los 84 diputados.

13. En circunstancias particulares puede, por el contrario, indicar el debilitamiento, y hasta el derrumbe, de un partido en cl nivel nacional. Un realineamiento clectoral en muchos municipios puede indicar un realineamiento electoral a nivel nacional.

14. Cfr. Artiga-González, 1996, Cap.V.

15. Se sabe que la fórmula Hare-restos mayores (que es la utilizada por el sistema electoral salvadoreño para elecciones parlamentarias) tiende a ser más proporcional que la fórmula D'Hondı, que produce desproporcionalidad al favorecer a los partidos grandes. Esta es la razón por la que escogimos esta última para hacer este ejercicio de comparación y así poder observar los efectos de la fórmula frente a los efeclos de la distribución de las circunscripciones sobre la proporcionalidad del sistema.

\section{Referencias bibliográficas.}

Artiga-González, Alvaro (1993). "Buscando la clave de las elecciones del 94", en Revisła Realidad económico-social, No.34, pp.467-479. San Salvador: Universidad Centroamericana "José Simeón Cañas".

Artiga-Gonźllez, Alvaro (1996). El Salvador: el ascenso electoral del partido ARENA y las "elecciones del siglo". (Tesis de Maestría en Ciencias Sociales). México: FLACSO.

CIDAl (1984). "Destapando la "caja negra". Condicionamientos

técnicos del proceso electoral 1984", en ECA-Estudios Centroomericanos, No.426-427, pp.197-218. San Salvador: Universidad Centroamericana "José Simeón Cañas".

Consejo Central de Elecciones, CCE (1982a). Elecciones para Asamblea Constisuyente. Resulrados parciales por departamento y por partido. San Salvador: folocopia.

Consejo Central de Elecciones, CCE (1985a). Elecciones para Asamblea Legislativa. (Resulrados al 8 de Mayo de 1985). San Salvador: fotocopia.

Consejo Central de Elecciones, CCE (1985b). Resultados provisionales, Elección de Alcaldes. San Salvador: fotocopia.

Consejo Central de Elecciones, CCE (1988a). Resullados por departamento, elecciones diputados, 20 de Marzo de 1988. San Salvador: fotocopia.

Consejo Central de Elecciones, CCE (1988b). Resullodos Elecciones Concejos Municipales, 20 de Marzo de 1988. San Salvador: fotocopia.

Consejo Central de Elecciones, CCE (1991a). Escrutinio final por departamento, elecciones para diputados 10 de Marzo de 1991. San Salvador: folocopia.

Consejo Central de Elecciones, CCE (194lb). Reporse de votación para Concejos Municipales, Elección 10 de Marzo de 1991. San Salvador: fotocopia.

Dirección General de Estadísticas y Censos (1992). V Censo de Población y Vivienda. San Salvador: Diskette. 
Editorial ECA (1982). "Las elecciones y la unidad nacional: diez tesis crílicas", en ECA-Estudios Centroamericonos, No.402, pp.233-258. San Salvador: Universidad Centroamericana "José Simeón Cañas".

Lijphart, Arend (1990). "The political consequences of electoral laws, 1945-85" en American Political Science Review, vol.84, No.2, pp.481-96.

Lijphart, Arend (1994). Electoral systems and party systems. New York: Oxford University Press.

Nohlen, Dieter (1993). Los sistemas electorales en América Latina y el debate sobre reforma electoral México: Universidad Nacional Autónoma de México.

Nohlen, Dieter (1994). Sistemas electorales y partidos políticos. México: Fondo de Cultura Económica.

Rae, Douglas (1967). The polisical consequences of electoral laws. New Haven: Yale University Press.

Sartori, Giovanni (1994). Ingenierla constitucional comparada. México: Fondo de Cultura Económica.

Taagepera, Rein \& Shugarh, Matthew (1989). Seats \& Vores. New Haven: Yale Universily Press.

Tribunal Supremo Electoral, TSE (1994a). Reporte de volación para diputados por departamento. Escrutinio final. San Salvador: folocopia.

Tribunal Supremo Electoral, TSE (1994b). Reporte de alcaldias obtenidas por cada partido o coalición, elección 20 de Marzo de 1994. Escrutinio final. San Salvador: folocopia.

Tribunal Supremo Electoral, TSE (1994c). Acta gencral nacional para elección de Concejos Municipales, 20 de Marzo de 1994. San Salvador: fotocopia.

Vanaclocha, Francisco (1990)."Procesos y sistemas electorales", en Pastor, Manuel (comp). Ciencin Políica, pp.233-302. Madrid: McGraw Hill. 